\section{La interdisciplinariedad en la enseñanza, un desafío para la escuela secundaria}

\author{
CAMILA CARLACHIANI ${ }^{(1)}$
}

(1) Profesora y licenciada en Ciencias de la Educación de la Facultad de Humanidades y Artes de la UnR. Maestranda de la Maestría en Educación de la UnLP. Profesora del Área del Curriculum de la carrera de Ciencias de la

Educación y de Curriculum y Didáctica del Ciclo de Formación Docente de la

Facultad de Humanidades y Artes de la UnR. Miembro pleno del Centro de Estudios e Investigaciones sobre el

Curriculum y la Didáctica (CEICYD) de la Facultad de Humanidades y Artes de la Unr. Miembro del

Equipo Técnico Pedagógico de la Dirección Provincial de Desarrollo Curricular y Relaciones Académicas del Ministerio de Educación de Santa Fe. camilacarlachiani@gmail.com
Palabras clave. enseñanza $\cdot$ interdisciplinariedad obligatoriedad · inclusión · educación secundaria

Resumen. El presente artículo constituye un avance de la tesis de maestría en curso titulada "Dispositivos curriculares en la escuela secundaria. Un análisis desde perspectivas poscríticas». Se estudia el desarrollo institucional del curriculum en la escuela secundaria en diálogo con los distintos niveles de decisión de políticas curriculares en clave de traducción (Casimiro Lopes et. al., 2013). Para ello, se propone como objetivo general analizar cómo se diseñan y desarrollan los dispositivos curriculares institucionales en las escuelas secundarias a partir de procesos de traducción de políticas curriculares. Con relación a los objetivos específicos: 1) Indagar de qué modo dialogan los dispositivos curriculares institucionales con las políticas curriculares de los niveles macro y meso en torno a la inclusión para la educación secundaria. 2) Identificar qué dispositivos curriculares diseñan y desarrollan las escuelas secundarias para garantizar la obligatoriedad y la inclusión. A partir de un primer acercamiento al campo, es posible vislumbrar que la interdisciplinariedad en la enseñanza y su vinculación con problemáticas sociales locales constituye un modo de abordaje institucional para promover la inclusión y la obligatoriedad en la educación secundaria favoreciendo aprendizajes socialmente significativos (Orozco Fuentes, 2006). 


\section{Interdisciplinarity in teaching: a challenge for secondary education}

Keywords. teaching $\cdot$ interdisciplinarity $\cdot$ compulsory education $\cdot$ inclusion $\cdot$ secondary school
1) to investigate how institutional curricular devices enter into dialogue with curricular policies of macro and meso levels about inclusion for secondary school; 2) to identify which curricular devices are designed and developed by secondary schools to guarantee compulsory education and inclusion. By taking a first approach to the field, it is possible to perceive that interdisciplinarity in teaching and its connection with local social problems constitute an institutional approach to promote inclusion and compulsory education in secondary school, favoring socially significant learning (Orozco Fuentes, 2006).

\section{Introducción}

El propósito de este escrito es analizar las prácticas que desafían el formato de la escuela secundaria a partir del desarrollo curricular institucional en clave de inclusión. Al interior de las escuelas se despliegan innumerables experiencias que asumen el desafío de transformar el formato escolar tradicional de la escuela secundaria con el fin de lograr la real inclusión de los jóvenes para quienes la escuela secundaria no fue pensada, esto es: el ingreso, la permanencia, el aprendizaje y el egreso en tiempo y forma.

A partir del año 2006, en Argentina, la sanción de la Ley de Educación Nacional (LEN) 26206 establece la obligatoriedad de la educación secundaria y legitima así un proceso que, desde la segunda mitad del siglo XX, amplió progresivamente el acceso a este nivel educativo a sectores históricamente excluidos. Sin embargo, la concreción de la obligatoriedad no es tarea sencilla. Si bien existen condicionamientos económicos, sociales, políticos y culturales para lograr el 
derecho a la educación secundaria de todos los jóvenes de nuestro país, también a nivel microinstitucional se encuentran sedimentos difíciles de conmover que obturan el mencionado acceso y permanencia. A pesar de ello, se generaron múltiples políticas curriculares en pos de la obligatoriedad.

Vale señalar que este arduo camino hacia la ampliación de derechos se da en diálogo con un intenso proceso supra nacional donde organismos internacionales como la UNESCO y países de América Latina comienzan a diseñar políticas que apuntan a la obligatoriedad de la educación secundaria. Así, la intención de este avance es analizar de qué modo las propuestas de enseñanza con interdisciplinariedad que se desarrollan en las escuelas secundarias, resultan de procesos de traducción (Casimiro Lopes et. al., 2013) desde el supra hasta el micronivel institucional (Stirling Network for Curriculum Studies, 2016). Metodológicamente, la investigación se desarrolla desde un enfoque interpretativo que implica la construcción de datos desde un estudio fenomenológico de la vida social (Taylor y Bodgan, 1987). Para ello, se realizan entrevistas a equipos directivos y grupos focales con profesores.

\section{Educación secundaria: disputa de sentidos}

En los últimos años, diversos discursos circulan en torno a los problemas de la educación secundaria. Unas veces tomando como eje de fuertes críticas a las características de los jóvenes, otras al trabajo de los profesores; lo cierto es que desde distintos sectores hay un acuerdo tácito en reconocer que la escuela secundaria está en crisis. Muchos autores explican esta situación en vinculación a las tensiones que se dan entre su propia matriz y lógica instituida y las demandas sociales que recibe actualmente.

Para comenzar a desarmar esta problemática es pertinente acudir a la categoría de cultura escolar desarrollada por Viñao (2002), la cual refiere a un conjunto de teorías, ideas, principios, normas, pautas, rituales, inercias, hábitos y prácticas (formas de hacer y pensar, mentalidades y comportamientos) sedimentadas a lo largo del tiempo en forma de tradiciones, regularidades y reglas de juego no puestas en entredicho, y compartidas por sus actores, en el seno de las instituciones educativas. Sus rasgos característicos serían la continuidad y 
persistencia, algo que permanece y que dura. Algo que las reformas no logran más que arañar superficialmente, que sobrevive a ellas, y que constituye un sedimento formado a lo largo del tiempo.

Si pensamos esta cuestión en el ámbito de la educación secundaria, observamos que muchas han sido las reformas educativas que intentaron generar algún movimiento en su formato escolar y en su cultura institucional. El señalado sedimento que poco a poco fue conformado un núcleo duro de conmover, opera constantemente en las prácticas que allí se desarrollan cuyos efectos principales son la exclusión y la expulsión hacia aquellos jóvenes que no se adaptan a sus reglas. En palabras de Nóbile:

El modelo institucional del bachillerato, con su curriculum enciclopédico, sus interacciones docente-alumno prefiguradas por medio de un reconocimiento a una autoridad basada en el conocimiento disciplinar y definida por nociones de respeto específicas, configuraron una cultura institucional a la que se adecúan mejor los jóvenes provenientes de sectores más afines a la cultura escolar, y que, por tanto, se acercan al patrón de alumno «normal» tradicionalmente esperado por el secundario, un joven dedicado plenamente a sus estudios, «respetuoso» y «responsable». (2016:122)

Esta expectativa hacia el tipo de estudiante que la escuela secundaria y los profesores poseen, se relaciona directamente con la matriz elitista, selectiva y propedéutica de su origen. Sin embargo, ello no obturó el avance hacia el desarrollo de políticas que extienden el acceso de este nivel educativo a sectores cada vez más amplios de la población, aunque sí presenta ciertos límites en la posibilidad de su concreción.

En las últimas décadas, la transformación más importante en la educación secundaria ha consistido en un cambio respecto de su definición viéndosela ahora como parte constitutiva de la educación fundamental que todo ciudadano debiera poseer, y ya no como una situación excepcional o de privilegio (Oficina Regional de Educación para América Latina y El Caribe, UNESCO, 2013). Es a partir de este proceso que diferentes países de América Latina han desarrollado nuevas políticas y normativas para la educación en general y la escuela secundaria en particular. Por ello, podríamos afirmar que los problemas en torno a la educación secundaria trascienden las fronteras de los países tornándose una problemática global. 


\section{Dimensión institucional}

Para referirse al formato escolar de la escuela secundaria como ese núcleo difícil de modificar a pesar de los cambios y transformaciones que se dan a nivel social, político, económico y cultural, Terigi (2008) elabora la noción del «trípode de hierro». Esta categoría ilustra la fortaleza casi inamovible de tres elementos clave que operan en la dinámica de la escuela secundaria y, muchas veces, se presentan como obstáculos a la hora de generar transformaciones en su interior. En palabras de la autora: «la escuela media se estructuró en torno a tres disposiciones básicas: la clasificación de los currículos, el principio de designación de profesores por especialidad y la organización del trabajo docente por horas de clase» (2008:64). Se trata de un patrón organizacional que es difícil de modificar, aunque no se trata de una tarea imposible. Acosta (2012) presenta algunas estrategias en pos de superar el modelo excluyente de la escuela secundaria: a) dentro de la oferta regular; b) a través de modelos alternativos de escolarización; c) a través de la revalorización de la orientación para el trabajo. De esta manera, el tipo de cambio que se requiere en el nivel secundario involucra una transformación profunda en el formato escolar que asumió históricamente el nivel (Terigi, 2008).

Terigi y Jacinto (2007) señalan que algunos caminos desarrollados desde las políticas y desde las escuelas para contribuir al mejoramiento de oportunidades para todos los que acceden a la educación secundaria se centraron primero en políticas compensatorias focalizadas conocidas como discriminación positiva; y más tarde, medidas transversales que, asumiendo la discriminación positiva, abarcan a todas las escuelas públicas. En nuestro país, existen numerosos intentos para generar cambios en este sentido, a través de diferentes políticas públicas que podemos enumerar en tres grandes grupos. En primer lugar, los Planes de Mejora Institucional a través de los cuales las escuelas identifican sus propias problemáticas y desarrollan líneas de acción teniendo como principal eje de trabajo el acompañamiento a las trayectorias escolares. En segundo lugar, la creación de instituciones alternativas de educación que introducen importantes modificaciones al formato tradicional de la escuela secundaria adaptándolo a las posibilidades de las poblaciones que atiende. Por último, los programas que permiten que quienes adeudan asignaturas del secundario, alcancen su titulación (Nóbile, 2016). 
Jacinto y Terigi (2007) alertan sobre las limitaciones que la estrategia centrada en la formulación de proyectos puede tener para impactar sobre la gestión institucional. Primeramente, señalan que los proyectos se centraron en las dificultades de los alumnos generando poca reflexión sobre las condiciones institucionales y los procesos de enseñanza. Luego, hay muchas variaciones en la forma en que los proyectos de una misma escuela conforman una propuesta institucional articulada o bien se constituye en una suma de proyectos aislados. Terigi (2015) sostiene que es fundamental hacer foco en las condiciones en que se produce la escolaridad secundaria, en particular las cuestiones vinculadas con el régimen académico, la cursada, las materias en bloque, la asistencia día por día. En síntesis, la posibilidad de definir trayectos más adecuados a las circunstancias de los estudiantes.

Pinkasz (2015) postula que muchas de las políticas educativas desarrolladas en los últimos años para la educación secundaria se centran en la escuela como unidad de desarrollo del cambio. Esto significa que son los equipos directivos y docentes los que asumen la responsabilidad de la mejora escolar y de los aprendizajes ya que son quienes conocen las necesidades de la comunidad.

Es posible vislumbrar entonces, a través de los aportes mencionados, que la dimensión institucional en torno a lo que la escuela ofrece, parece ser un punto clave a la hora de revisar la enseñanza y garantizar la obligatoriedad y la inclusión en el nivel secundario. En relación con ambas nociones, Bracchi (2014) postula que la obligatoriedad es una batalla cultural que impone al Estado la responsabilidad de generar condiciones materiales y simbólicas para que todos los jóvenes accedan, permanezcan, aprendan y egresen de la escuela secundaria. Por otro lado, entiende a la inclusión como categoría política que se piensa desde un enfoque de derechos que concibe a los adolescentes y jóvenes como sujetos plenos, con capacidad de decisión, con derechos y responsabilidades, y con potencialidad para ejercer y construir ciudadanía.

\section{Dimensión curricular y la enseñanza}

El curriculum es uno de los elementos que conforman el trípode de hierro de la escuela secundaria por ser enciclopedista y, en consecuencia, anacrónico 
(Terigi, 2012). Además de esto, Jacinto y Terigi (2007) sistematizan otros de los problemas y críticas que históricamente posee el curriculum de la escuela secundaria. La fragmentación en numerosas asignaturas (siempre más de diez), la inexistencia de alternativas para la elección personal, la relegación de los intereses de los adolescentes y de los problemas del mundo contemporáneo, la ausencia de una orientación hacia el desarrollo de capacidades intelectuales y prácticas básicas. Se cuestiona así, al curriculum, por sus efectos de una formación de escasa relevancia social, en términos de la contribución de la escuela a la ciudadanía, la ampliación al acceso de los bienes culturales, y la inserción en actividades productivas como también la continuidad hacia los estudios superiores.

Sin embargo, ante este crítico panorama, el curriculum se constituye especialmente en eje de análisis, ya que, según lo que plantean diversos autores, es una dimensión en la que se podría generar algún movimiento que resulte apropiado en relación a los aprendizajes de los estudiantes. Para ello, Jacinto y Terigi (2007) se preguntan qué cambios relevantes soporta la actual estructura del curriculum sin someterla a grandes transformaciones. Las autoras postulan que es posible introducir en el curriculum nuevos componentes que permitan incorporar temáticas ausentes en los planes de estudio vigentes, y que abran algunas posibilidades de elegir en la formación de los estudiantes así como revisar la relevancia de los contenidos.

Otro de los modos a través de los cuáles sería posible ofrecer espacios que otorguen nuevos sentidos al estar, habitar, transitar la escuela secundaria, es instaurar en la enseñanza el criterio de contemporaneidad (Gurevich, 2009). Se trata de ofrecer a los estudiantes tramas de sentido entre las vidas individuales y el contexto de la época teniendo en cuenta la materialidad de los nuevos recursos y objetos tecnológicos; $y$ las subjetividades particulares que se producen a través de los múltiples símbolos y representaciones presentes en la vida cotidiana de nuestros días. En palabras de la autora:

si pensamos las culturas en plural, los territorios en plural, las sociedades en plural, hemos de transmitir a los estudiantes múltiples y diversas lógicas, preferencias y puntos de vista de los sujetos individuales y colectivos que permanentemente modelan y construyen las diferentes textualidades del mundo. (Gurevich:25) 
En relación con esto, es factible acudir a los aportes de José de la Luz Sánchez Tepatzi (2011), quien analiza los significados de valor que los estudiantes asignan a la escuela secundaria mediante la experiencia escolar. Para ello, señala cómo se traduce el curriculum formal en una experiencia escolar relevante y cómo influye tal significado en la permanencia escolar. Una de las claves para atender a las problemáticas esbozadas por estos autores, radica en incorporar la interdisciplinariedad como modo de superar la fragmentación de los saberes y, a la vez, romper los muros de la escuela como institución cerrada (Torres Santomé, 1994).

\section{Las políticas curriculares y sus traducciones}

En la transición acontecida entre finales del siglo XX y principios de siglo XXI asistimos a un cambio económico, social, político y cultural que disloca las estructuras a partir de las cuales la modernidad se erigió. Frente a la incredulidad hacia los metarrelatos, Lyotard se pregunta: ¿dónde puede residir la legitimación después de estos? Y formula la siguiente hipótesis: «el saber cambia de estatuto al mismo tiempo que las sociedades entran en la edad llamada posindustrial y las culturas en la edad Ilamada posmoderna» (1991:6). De este modo, el saber científico ya no goza de una absoluta hegemonía sino que es un modo de saber más, entre otros, tales como los saberes culturales, generacionales, experienciales, etcétera.

Frente a este escenario es necesario considerar a las políticas curriculares como aquellos discursos que, mediante traducciones (Casimiro Lopes et. al. , 2013), generan y promueven prácticas de enseñanza y aprendizaje desde el supra hasta el micro nivel (Stirling Network for Curriculum Studies, 2016). Vale señalar, como afirman Casimiro Lopes et. al., que toda traducción es una producción original, un acontecimiento. Siguiendo a Badiou, «un acontecimiento es algo que hace aparecer cierta posibilidad que era invisible o incluso impensable. Un acontecimiento no es por sí mismo una creación de realidad; es creación de una posibilidad»(2013:21). Es importante entonces, analizar qué acontecimientos en términos de posibilidades en la educación secundaria se dan en el 
micro nivel de las políticas curriculares para diseñar propuestas de enseñanza interdisciplinarias, reconociendo los juegos de traducción antes mencionados. El supra nivel se compone de las políticas educativas y curriculares que emanan de organismos internacionales como pueden ser la UNESCO, el Banco Mundial, la OCDE, etc. Se trata de organizaciones que superan los límites de los Estados y diseñan políticas a nivel mundial para la educación. Luego, el macro nivel, corresponde a los ámbitos de diseño y decisión política en el marco de cada Estado nacional o país. El meso nivel se vincula a las jurisdicciones que dentro de un Estado organizan políticamente el territorio. En Argentina, por ejemplo, el meso nivel corresponde a las jurisdicciones provinciales. Continuando este recorrido del nivel supra al nano, encontramos el nivel micro, que se refiere a las decisiones y acciones que se llevan adelante al interior de las instituciones educativas. Por último, en el nivel nano nos referimos al aula, como el ámbito concreto en el que las decisiones políticas se materializan a través del trabajo concreto de los profesores (Carlachiani, 2017).

Para dar tratamiento a la enseñanza con interdisciplinariedad como un desafío frente al patrón organizacional de la escuela secundaria, nos centramos en el micro nivel para considerar la producción de dispositivos curriculares como aquellas prácticas que propician la construcción y la apropiación de saberes por parte de los estudiantes. La noción de dispositivos curriculares es utilizada como categoría intermedia (Buenfil Burgos, 2007). Su significado radica en ser una figura de intelección de alcance intermedio, una imagen analítica que ensambla la generalidad teórica con la particularidad histórica. Es una herramienta analítica que depende de su relación con el objeto en construcción y no tiene una positividad o autonomía propias. En el marco de esta investigación, los dispositivos curriculares aluden al modo material en que la escuela secundaria garantiza la inclusión. Se trata de construcciones discursivas que producen prácticas donde los estudiantes son escuchados y sus intereses son alojados en la propuesta escolar. Los dispositivos curriculares amarran saberes, relaciones de poder, y producen subjetividades. Articulan lo dicho y lo no dicho que también es discursivo. Son prácticas que perforan el mandato escolar elitista, academicista, enciclopedista y propedéutico de la escuela secundaria porque alteran el disciplinamiento de los cuerpos permitiendo el agenciamiento de los sujetos. 
(1) Resolución CFE 84/o9 «Lineamientos Políticos y Estratégicos de la Educación

Secundaria Obligatoria»; Resolución CFE 88/o9 «Institucionalidad y Fortalecimiento de la Educación Secundaria Obligatoria: Planes Jurisdiccionales y Planes de Mejora Institucional (PMI)»; Resolución CFE 93/o9 «Orientaciones para la organización pedagógica e institucional de la Escuela

Secundaria Obligatoria»; Resolución CFE I03/Io «Pautas Federales para la movilidad estudiantil en la Educación Obligatoria»; Resolución CFE IO2/IO «Propuestas de inclusión y/o regularización de trayectorias escolares en la educación secundaria».
Atravesados por la tensión global/local, su configuración es local y particular puesto que cada escuela arma dispositivos curriculares de acuerdo a su contexto, recursos, necesidades, objetivos, modos de ser de los sujetos que la habitan. A la vez, desde un punto de vista global, su puesta en marcha responde al imperativo de inclusión social a partir de la obligatoriedad de la educación secundaria y el sostenimiento de la educación como un derecho.

\section{La enseñanza con interdisciplinariedad en el micronivel}

Arribamos a este apartado con el propósito vislumbrar cuáles son los dispositivos que a nivel escolar se llevan adelante como traducciones de las macro y meso políticas curriculares en relación a la enseñanza con interdisciplinariedad. En primer lugar, cabe señalar que tomamos como documento fundamental del macro nivel en Argentina a la LEN 26206/06 que establece la obligatoriedad de la educación secundaria basándose en la educación como un derecho y el conocimiento como un bien público. Con este antecedente normativo, diferentes resoluciones del Consejo Federal de Educación (CFE) sintetizan y dan integralidad a las políticas con el fin de alcanzar el objetivo de la obligatoriedad. (1) Dentro de este marco normativo, Ios PMI (Planes de Mejora Institucional) se convierten en un instrumento para que la propuesta educativa del nivel secundario se despliegue en múltiples concreciones con arraigo y sentido institucional. Este programa consiste en una política pública nacional a través de la cual el Estado transfiere recursos a las provincias y a las escuelas para que instrumenten modificaciones al modelo tradicional de la escuela secundaria incorporando variaciones en su organización académica (Pinkasz, 2015) con el fin de acompañar a las trayectorias de los estudiantes que se encuentran en situaciones de riesgo escolar. Así, se proponen diversas acciones relacionadas a: organizar propuestas de enseñanza interdisciplinar; generar propuestas sociocomunitarias; actividades complementarias. También se sugiere la alternativa de ofrecer Tutorías Académicas (clases de apoyo opcionales no curricularizadas) para aquellos estudiantes que necesitan recuperar aprendizajes, realizar acompañamiento, etc. Para llevar adelante esta tarea, el Estado nacional transfiere 
fondos a las provincias con el fin de formar equipos técnicos territoriales y, además, contratar profesores (tutores académicos) que se suman al equipo institucional y trabajan de forma articulada con los docentes de cátedra. El programa se inició en 2010 con el $50 \%$ de las escuelas de todo el país, y en 2011 se extendió al resto.

En un trabajo de Terigi et. al (2013), se plasman algunas características interesantes del PMI y se afirma que el mismo presenta alguna potencialidad para promover cambios en la matriz organizacional tradicional ya que amplía el tiempo de ciertos profesores en la escuela permitiendo que se desempeñen nuevos roles (por ejemplo, tutores); supone una posibilidad para ampliar el tiempo en la escuela por parte del estudiante; promueve la incorporación de nuevos docentes en la escuela aunque sea en modo transitorio; permite un trabajo más personalizado con los estudiantes que asisten a las clases de apoyo. No obstante, uno de los riesgos que señala se vincula a que la innovación que promueve el PMI opera en el marco de las condiciones de escolarización de la escuela secundaria estándar, y por esta razón es posible que se diluya el propósito transformador de la propuesta.

Por otra parte, en el meso nivel, se desarrolla en la provincia de Santa Fe, a partir del año 2016, el programa Secundario Completo cuyo principal propósito es el acompañamiento integral de las trayectorias escolares de los/las estudiantes con el fin de garantizar la obligatoriedad del nivel y con ello, el derecho a la educación. Se trabaja en el diseño de trayectorias artesanales donde participan docentes, familias, y estudiantes con el fin de que éstos ingresen, permanezcan, aprendan y egresen de la escuela secundaria. Se espera así la construcción en forma colectiva de aprendizajes cuya relevancia social les otorgue una educación de calidad. En este marco de diseñan y desarrollan los denominados Espacios de Acompañamiento. Refieren a aquellos lugares y tiempos dentro y fuera de la escuela donde los estudiantes transitan y desarrollan sus trayectorias. El aula, la biblioteca, el patio, instancias virtuales, la sala de usos múltiples, el salón de música, artes, idiomas, el cursado en turno o contraturno. Son espacios y tiempos donde la enseñanza y el aprendizaje se llevan adelante a través de diferentes propuestas pedagógicas, con distintos recursos para realizar actividades, utilizando múltiples lenguajes (PSC, 2016). 
Ahora bien, cabe preguntarse entonces, ¿cómo se traducen estas políticas macro y meso al nivel micro institucional? En la escuela tomada como unidad de análisis es posible vislumbrar rasgos vinculados a: entender la educación y la obligatoriedad de la secundaria como un derecho; generar espacios y tiempos que permitan el recorrido diverso de las trayectorias escolares; promover la enseñanza y el aprendizaje con interdisciplinariedad; potenciar la relación de la escuela con la comunidad y demás instituciones sociales.

Al momento de describir cómo es esta escuela, una de las entrevistadas, miembro del equipo directivo de la institución, afirma:

Yo la podría describir con una palabra que es inclusiva. Que hacemos todo lo posible para que los chicos ingresen y permanezcan dentro de la institución. Se les hace un seguimiento. Contamos con tutores, facilitadores de primero a quinto año. (...) Cada curso tiene un profesor que es su referente. $Y$ ese profesor conoce a cada alumno y su realidad familiar. (Entrevista 1)

Esto mismo se plasma en el proyecto institucional: «La escuela asume la responsabilidad de incluirlos, asegurando su ingreso y permanencia. El desafío es garantizarles aprendizajes con calidad». Para ello, se despliegan innumerables estrategias que son relatadas en las entrevistas: «los estudiantes vienen con muchas carencias en cuanto a no dialogar con sus familias. Y acá en la escuela encuentran un lugar de escucha. Eso hace que los chicos puedan permanecer y tengan confianza dentro de la escuela» (Entrevista 1). La apertura de la escuela hacia la voz de los estudiantes es un rasgo importante a la hora de fortalecer el vínculo con ellos. Por otra parte, el trabajo de los profesores constituye otra base fundamental. Así lo manifiesta la vicedirectora: «los docentes tienen mucha predisposición y lo vemos en las plenarias y con cada proyecto que se presenta desde la escuela inmediatamente se ponen a trabajar». Además, la comunicación entre los actores institucionales también parece ser un punto clave de la dimensión institucional.

Tenemos una fluida comunicación en el equipo directivo. Nos encontramos una vez por semana. Es nuestro momento de encuentro y de reunión. La asesora pedagógica también forma parte del equipo de gestión. Más allá de los turnos, la escuela es una. Y nosotras estamos interiorizadas de todo lo que ocurre en la escuela. Se trabaja en equipo. Nosotros como equipo de 
gestión y los docentes también. Hay mucho trabajo en equipo. Creo que es la única forma: colaboración y diálogo.

Otro aspecto importante además de la escucha, la comunicación y el trabajo en equipo es la dimensión curricular y de la enseñanza. Podría afirmarse que en esta institución es una de las líneas centrales ya que es imposible trabajar en pos de la inclusión sin tener en cuenta la calidad educativa, entendiendo por ésta a la relevancia de los saberes que la escuela enseña. «Para la enseñanza utilizamos los NIC, Núcleos Interdisciplinarios de Contenidos. Entonces en diferentes materias se toma un tema determinado y se trabaja interdisciplinariamente. Se hacen proyectos, talleres, exposiciones. Los chicos son los que más potencial tienen. Muchas veces nos asombramos de los resultados». Los NIC son una política educativa del meso nivel que apunta al fortalecimiento del desarrollo curricular institucional. Se trata de una propuesta metodológica para la enseñanza con interdisciplinariedad a partir de problemáticas sociales entendidas como acontecimiento. Los NIC son un modo no convencional de pensar el curriculum. Su armado se concreta partiendo de problemáticas sociales y culturales situadas entendidas como acontecimiento; y su diseño, puesta en acción y evaluación se realiza desde una perspectiva interdisciplinaria de saberes a ser enseñados (científicos, artísticos, tecnológicos, culturales) que se configuran desde una lógica interdisciplinar. Se propone tomar como punto de partida problemáticas regionales propias de los contextos que habitan los/las estudiantes que conduzcan al abordaje de contenidos escolares de los espacios curriculares que integran la propuesta educativa institucional. Se espera que desde cada espacio curricular, las disciplinas realicen aportes al abordaje de la problemática social planteada constituyendo NIC y posibilitando así el debate, el intercambio y la construcción de sentidos, lo cual permitiría la invención de respuestas creativas e innovadoras por parte de los/las estudiantes. Un ejemplo de ello, es el trabajo interdisciplinario realizado por asignaturas como Formación Ética y Ciudadana; Lengua y Literatura e Historia donde se trabajaron problemas de discriminación y violencia de género a partir del «Ni una menos». Los estudiantes de segundo año realizaron una muestra fotográfica denominada «Los prejuicios de envenenan el juicio» donde se desarman ciertos prejuicios sociales. 
En el proyecto institucional de la escuela se describe como una estrategia clave la necesidad de conformar una Comunidad de Práctica. Citando a Wenger (1998), se explicita que una comunidad de práctica es más que un trabajo en equipo, es un grupo que se constituye para desarrollar un conocimiento especializado, compartir experiencias basados en la reflexión conjunta. El resultado es el enriquecimiento profesional para poder mejorar las prácticas. La estrategia comprende la formación de la comunidad de práctica, que hará un recorrido en revisión de prácticas pedagógicas mediante autoformación y coformación. Se incorpora un plan de observación entre pares y espacios de reflexión sobre estas prácticas para trabajar en el rediseño de propuestas de aula. Vale destacar que en esta comunidad no se involucra solamente al equipo directivo y a los profesores sino también a otros actores institucionales que comparten la tarea tales como preceptores, secretarios, bibliotecarios.

Como resultados del trabajo institucional en equipo, una de las entrevistadas manifiesta: "Los resultados que estamos teniendo últimamente es por el trabajo de muchos años. La matrícula se mantiene desde el ingreso al egreso (...) la escuela inclusiva va más allá de lo pedagógico». Vemos en este último fragmento condensadas múltiples dimensiones y estrategias que la escuela pone en marcha y entendemos — en este trabajo — como dispositivos curriculares. Estos no se conforman solo de los saberes de los profesores, también se involucra la voz de los estudiantes, la conducción del equipo directivo, la colaboración de preceptores, secretarios y bibliotecarios, la relación permanente con las familias. El micronivel institucional se constituye así en el espacio y tiempo que hace posible la concreción de las políticas curriculares del macro y meso nivel. Es en lo particular, local que no pierde de vista lo global, donde la inclusión es un hecho y el derecho a la educación una realidad.

\section{A modo de reflexión}

El conocimiento acerca de los dispositivos curriculares desplegados en la institución analizada a partir de un primer acercamiento al campo permite esbozar algunas reflexiones preliminares. En primer lugar, la enseñanza con interdisciplinariedad se presenta como una práctica que desafía el trípode de hierro de 
la escuela secundaria. Es posible vislumbrar que, a pesar de ciertos límites y condicionamientos, el trabajo en equipo y el desarrollo de una comunidad de práctica, favorecen ciertos procesos de cambio. De este modo, podría afirmarse que, elaborar propuestas de enseñanza interdisciplinarias que dialogan con la oferta regular (Acosta, 2012) —es decir, con el diseño curricular vigente-, propicia la construcción de aprendizajes socialmente significativos (Orozco Fuentes, 2006) que fortalecen la vinculación de la escuela con la comunidad. Al mismo tiempo, la incorporación de criterios de contemporaneidad en la enseñanza (Gurevich, 2009) permite a los estudiantes tener una comprensión integral de la realidad superando visiones fragmentadas y sesgadas.

Es posible vislumbrar el modo en que los diferentes niveles de políticas curriculares realizan traducciones en torno a la enseñanza con interdisciplinariedad. Observamos esta cuestión tanto en la LEN como en los documentos del PMI a nivel macro. Luego, vimos cómo los NIC realizaron su propia traducción mediante una política curricular jurisdiccional, y finalmente, de qué manera esto se tradujo en un proyecto institucional llamado «Los prejuicios te envenenan el juicio».

Este recorrido nos permite afirmar, siguiendo a Torres Santomé (1994), que la interdisciplinariedad es fundamentalmente un proceso y una filosofía de trabajo que se pone en acción a la hora de enfrentarse a los problemas y cuestiones que preocupan a cada sociedad. Se trata de un objetivo nunca alcanzado por completo y de ahí que deba ser permanentemente buscado. Es ante todo una práctica. Su perfectibilidad se lleva a cabo en la medida en que se hacen experiencias reales de trabajo en equipo, se ejercitan sus posibilidades, problemas y limitaciones.

\section{Referencias bibliográficas}

Acosta, F. (2012). La escuela secundaria argentina en perspectiva histórica y comparada: modelos institucionales y desgranamiento durante el siglo XX. Cuadernos de historia de la educación, 11(1), 131-144. Badiou, A. (2013). La filosofía y el acontecimiento. Buenos Aires: Amorrortu.

Bracchi, C. (2014). La escuela secundaria: del paradigma de la selección al de la obligatoriedad. Novedades Educativas, (283). Buenos Aires: Noveduc.

Buenfil Burgos, R.N. (noviembre de 2007). La categoría intermedia. Ponencia para el IX Congreso Nacional de Investigación Educativa. Mérida: Yuc. 
Carlachiani, C. (2017). Políticas públicas para la educación secundaria: entre la obligatoriedad y la inclusión. Periferia. Educacao, cultura y comunicacao, 9(1, jan.-jun.), 383-405.

Casimiro Lopes, A.; Virgilio Rodriguez Da Cunha, E. y Costa, H. (2013). Da recontextualização à tradução: investigando políticas de currículo. Currículo sem Fronteiras, 13(3, set./dez.), 392-410.

Gurevich, R. (2009). Educar en tiempos contemporáneos: una práctica social situada. Propuesta Educativa, 18.2(32), 23-31.

Jacinto, C. (2012). Consideraciones sobre estrategias de inclusión con calidad en la escuela secundaria. SITEAL.

Lyotard, J.F. (1987). La condición postmoderna. Informe sobre el saber. Buenos Aires: Red Editorial Iberoamericana.

Morelli, S. (2016). Núcleos interdisciplinarios de contenidos. La educación en acontecimientos. Rosario: Homo Sapiens.

Nóbile, M. (julio de 2016). La escuela secundaria obligatoria en Argentina: desafíos pendientes para la integración de todos los jóvenes. Última década, (44), 109-131. Proyecto juventudes.

Orozco Fuentes, B. (2006). Aprendizajes socialmente significativos: en dialogo y tensión con los discursos del aprendizaje y las competencias en educación. Ponencia presentada en el Foro: 50 años del Colegio de Pedagogía. Facultad de Filosofía y Letras, Universidad Nacional Autónoma de México.

Pinkasz, D. (2015). Sobre la escuela como eje del cambio de las políticas de educación secundaria en la Argentina. Dossier revista Propuesta Educativa, 24.2(44), pp. 8-23.
Sánchez Tepatzi, J. (2011). ¿Cómo se traduce el curriculum formal en una experiencia formal relevante para los alumnos de una escuela telesecundaria de México? Actualidades investigativas en educación, 11(2), 1-22. San Pedro de Montes de Oca, Costa Rica.

Taylor, S.J. y Bodgan, R. (1987). Introducción a los métodos cualitativos de investigación. La búsqueda de significados. Barcelona: Paidós.

Terigi, F. y Jacinto, C. (2007). ¿Qué hacer ante las desigualdades en la educación secundaria?: aportes de la experiencia latinoamericana. Buenos Aires: UNESCO-IIPE Santillana.

Terigi, F. (2008). Los cambios en el formato de la escuela secundaria argentina: por qué son necesarios, por qué son tan difíciles. Propuesta Educativa, 15.1(29), 63-71.

- (2012). Sobre la cuestión curricular en la educación secundaria. En Tenti Fanfani (Coord.). La escolarización de los adolescentes: desafíos culturales, pedagógicos y de política educativa. Buenos Aires: UNESCO-IIPE.

--- (2015). Conversación con Flavia Terigi. La inclusión en la escuela media ante la persistencia del modelo escolar tradicional. En Diálogos del SITEAL, OEI, UNESCO.

Torres Santomé, J. (1994). Globalización e interdisciplinariedad: el curriculum integrado. Madrid: Morata. UNESCO. Oficina Regional de Educación para América Latina y El Caribe (2013). Situación educativa de América Latina y El Caribe: hacia la educación de calidad para todos al 2015. Santiago de Chile: Ediciones del Imbunche.

Viñao, A. (2002). Sistemas educativos, culturas escolares y reformas. Continuidades y cambios. Madrid: Morata. 